\title{
References
}

1 Hansell DM, Bankier AA, MacMahon H, et al. Fleischner Society: glossary of terms for thoracic imaging. Radiology 2008; 246: 697-722.

2 Cordier J-F, Johnson SR. Multiple cystic lung diseases. In: J-F Cordier, ed. Orphan Lung Diseases (ERS Monograph). Sheffield, European Respiratory Society, 2011; pp. 46-83.

3 Johnson SR, Cordier J-F, Lazor R, et al. European Respiratory Society guidelines for the diagnosis and management of lymphangioleiomyomatosis. Eur Respir J 2010; 35: 14-26.

4 Bonelli FS, Hartman TE, Swensen SJ, et al. Accuracy of high-resolution CT in diagnosing lung diseases. AJR Am J Roentgenol 1998; 170: 1507-1512.

5 Koyama M, Johkoh T, Honda O, et al. Chronic cystic lung disease: diagnostic accuracy of high-resolution CT in 92 patients. AJR Am J Roentgenol 2003; 180: 827-835.

6 Henske EP, McCormack FX. Lymphangioleiomyomatosis-a wolf in sheep's clothing. J Clin Invest 2012; 122: 3807-3816.

7 McCormack FX, Inoue Y, Moss J, et al. Efficacy and safety of sirolimus in lymphangioleiomyomatosis. $N$ Engl J Med 2011; 364: 1595-1606.

8 Young LR, Vandyke R, Gulleman PM, et al. Serum vascular endothelial growth factor-D prospectively distinguishes lymphangioleiomyomatosis from other diseases. Chest 2010; 138: 674-681.

9 Young L, Lee HS, Inoue Y, et al. Serum VEGF-D a concentration as a biomarker of lymphangioleiomyomatosis severity and treatment response: a prospective analysis of the Multicenter International Lymphangioleiomyomatosis Efficacy of Sirolimus (MILES) trial. Lancet Respir Med 2013; 1: 445-452.

10 Menko FH, van Steensel MA, Giraud S, et al. Birt-Hogg-Dubé syndrome: diagnosis and management. Lancet Oncol 2009; 10: 1199-1206.

\section{Blood stem cell transplantation to treat cystic lung light chain deposition disease}

\author{
To the Editor:
}

Light chain deposition disease (LCDD) is a rare disease resulting from non-amyloid immunoglobulin (Ig) light chain deposition in tissue. In systemic LCDD, plasma cell dyscrasia is common and renal involvement is almost always present, sometimes with damage to other organ systems (cardiac, hepatic and neurological systems). LCDD can be limited to the lungs, presenting as multiple cystic lung disease, nodules or bronchiectasis. Cystic lung related to LCDD (CL-LCDD) was recently described in patients referred for lung transplantation to treat end-stage multiple cystic lung disease [1]. In the reported cases, blood and bone marrow examinations did not reveal clonal plasma cell proliferation. Here we report the first case of CL-LCDD revealing a B-cell extrapulmonary lymphoproliferative disorder and the results of treatment for the underlying haematological disease with autologous peripheral blood stem cell transplantation during the CL-LCDD. Despite respiratory insufficiency, lung transplantation was actually not considered because of the underlying disease.

A 37-year-old Caucasian female was referred to Toulouse University Hospital (Toulouse, France) with cough, dyspnoea and fever of 5 months duration. The patient had sinusitis and bronchitis in infancy, and a close family member had been treated for lymphoma. She had recently quit smoking (smoking history: 10 pack-years). Physical examination revealed only one small indurated cervical lymphadenopathy. Pulmonary function tests showed an obstructive pattern with profound hypoxaemia and altered diffusing capacity of the lung for carbon monoxide: forced expiratory volume in $1 \mathrm{~s}$ (FEV1) was $1700 \mathrm{~mL}$ (56\% predicted), vital capacity was $2500 \mathrm{~mL}$ ( $73 \%$ predicted), total lung capacity was $103 \%$ predicted, diffusing capacity of the lung for carbon monoxide corrected for haemoglobin was $47 \%$ predicted and the partial pressure of oxygen in arterial blood was $42 \mathrm{mmHg}$. A computed tomography (CT) scan revealed diffuse thin-walled cystic formations associated with segmental atelectasis, emphysematous-like changes, bilateral bronchiectasis, and calcified hilar and subcarinal lymph nodes (fig. 1a). Positron emission tomography (PET)-CT revealed minimal uptake in the subcarinal lymph node. Histology of endobronchial biopsies revealed typical LCDD characterised by an inflammatory infiltrate with extracellular amorphous eosinophilic deposits (fig. 1b), negative Congo-red staining and no birefringence under polarised light. Immunofluorescence assays of frozen tissue revealed intense labelling for anti- $\kappa$ light chain antibody. The diagnosis was CL-LCDD.

An extensive search for extrapulmonary organ involvement was negative. Serum densitometry revealed a small monoclonal $\operatorname{IgM} \kappa$ peak $\left(0.29 \mathrm{~g} \cdot \mathrm{L}^{-1}\right)$, with associated hypogammaglobulinaemia $\left(6.64 \mathrm{~g} \cdot \mathrm{L}^{-1}\right)$. 

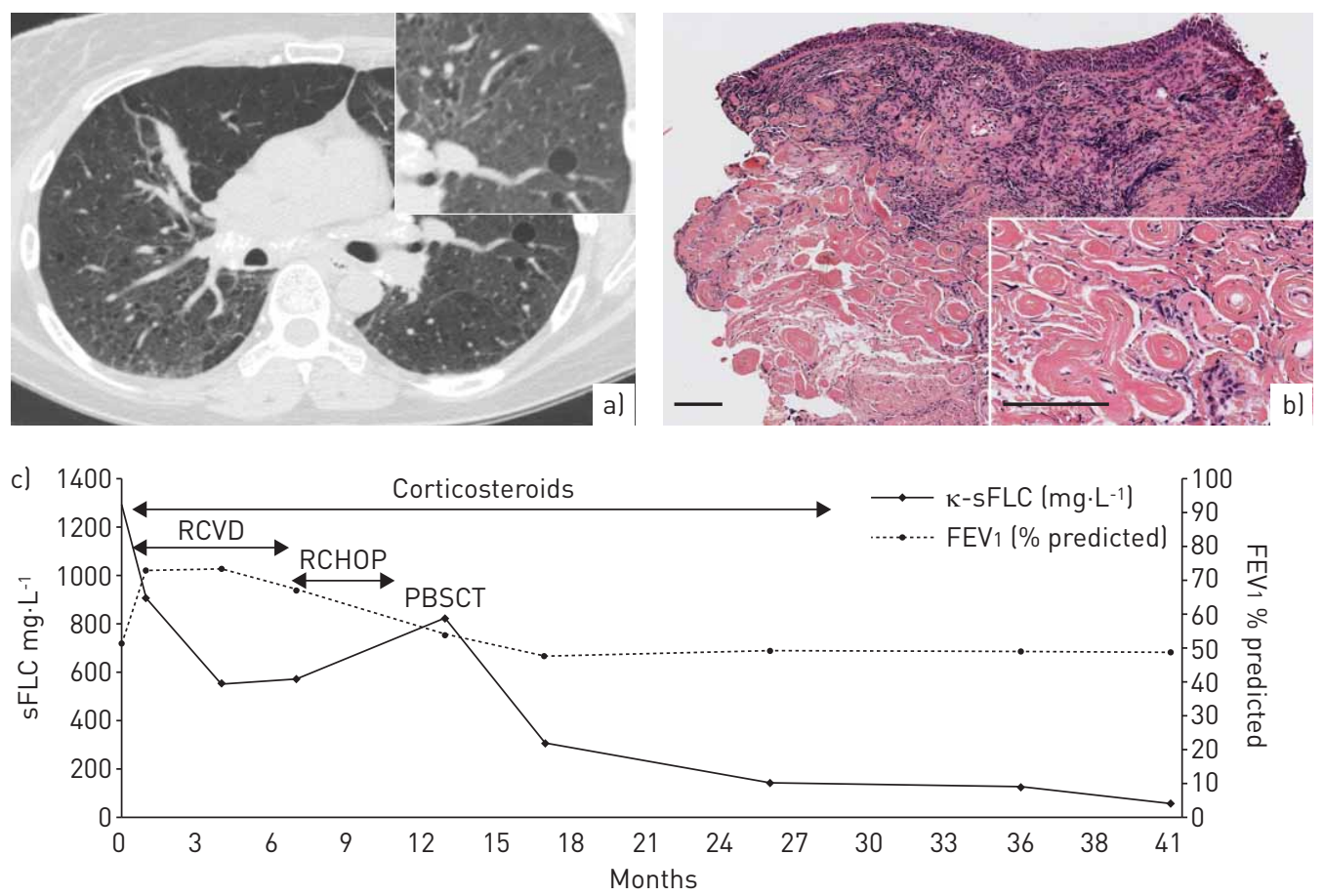

FIGURE 1 a) CT image at diagnosis of light chain deposition disease showing multiple cystic lung disease, bronchiectasis and emphysematous-like changes. b) Histology with haematoxylin-eosin-saffron staining of bronchial biopsies showing chronic inflammatory infiltrates and extracellular amorphous eosinophilic deposits, mainly perivascular (inset: higher magnification). Scale bars $=100 \mu \mathrm{m}$. c) Forced expiratory volume in $1 \mathrm{~s}$ (FEV 1 ) and level of $\kappa$ serum free light chains ( $\kappa-\mathrm{s} F L C$ ) with treatment. RCVD: rituximab, cyclophosphamide, bortezomib, dexamethasone; RCHOP: rituximab, cyclophosphamide, hydroxydaunorubicin, vincristine, prednisone; PBSCT: autologous peripheral blood stem-cell transplantation.

No cryoglobulinaemia was found. The level of $\kappa$ serum free light chains (sFLC) and $\kappa / \lambda$ sFLC ratio were increased to $1290 \mathrm{mg} \cdot \mathrm{L}^{-1}$ (normal range: $3.3-19.4 \mathrm{mg} \cdot \mathrm{L}^{-1}$ ) and 156.17 (normal range: 0.26-1.65), respectively. Bone marrow cytology revealed no signs of a plasma cell disorder. A bone marrow biopsy demonstrated a lymphoplasmacytic lymphoma with positive staining for IgM $\kappa$. Mediastinoscopy revealed lymphadenopathy with infiltration by the lymphoma and by light chain deposits without amyloidosis.

The treatment course is in figure $1 \mathrm{c}$. Corticosteroids at $1 \mathrm{mg} \cdot \mathrm{kg}^{-1}$ improved the cough, dyspnoea, FEV 1 and $\kappa$-sFLC level. The patient then underwent six chemotherapy cycles with bortezomib, cyclophosphamide, dexamethasone and rituximab. The first three cycles were effective, but then clinical deterioration was observed. The CT findings were not modified. Second-line chemotherapy was implemented with rituximab, cyclophosphamide, hydroxydaunorubicin, vincristine and prednisolone. Hypoxaemia, CT findings and $\kappa$-sFLC level worsened after two cycles. The patient underwent autologous peripheral blood stem cell transplantation after high-dose melphalan $\left(200 \mathrm{mg} \cdot \mathrm{m}^{-2}\right)$, which was well tolerated, with a rapid improvement in sFLC level. Although CT revealed a progression of emphysematous-like lesions, at 30 months, the patient was still alive. Long-term oxygen therapy was limited to deambulation, the $\kappa$-sFLC level was reduced to $4.3 \%$ of the initial level $\left(55 \mathrm{mg} \cdot \mathrm{L}^{-1}\right)$ and $\mathrm{FEV} 1$ was stable (50\% predicted).

Pulmonary LCDD is classically described in two clinic-radiological patterns: nodular and diffuse, including CL-LCDD, apart from pulmonary disorders present in systemic LCDD. A third presentation involves the airways $[2,3]$. In nodular disease, patients are usually asymptomatic or have moderate respiratory symptoms (e.g. cough and dyspnoea) with multiple nodules. Association with a lymphoproliferative disorder is common, e.g. gammopathy of undetermined significance, lymphoplasmacytic lymphoma, mucosa-associated lymphoid tissue (MALT) lymphoma or chronic lymphocytic leukaemia (CLL) [4]. The disease does not progress to chronic respiratory insufficiency, and the prognosis is linked to malignant B-cell dyscrasia. CL-LCDD should be considered among the causes of multiple cystic lung diseases, especially with nodes or bronchiectasis. According to this case and Colombat et al. [2], the level of sFLC and endobronchial biopsies using tissue frozen during a flexible bronchoscopy are useful for diagnosis.

CL-LCDD has been reported in five cases $[1,2,5]$. The patients were predominantly female, were 20-64 years-old and were light or nonsmokers. Four patients showed progression to respiratory failure; one, in the absence of medical care, to death. No monoclonal peaks were detected. Two patients showed increased 
$\kappa / \lambda$ sFLC ratio [1,5]. Bone marrow did not show plasmacytic proliferation. Colombat et al. [6] found a monoclonal population of B-cells in every pulmonary tissue sample but not in blood samples. The authors did not exclude that CL-LCDD could be a form of pulmonary MALT lymphoma without the histological diagnostic criteria of the World Health Organization Lymphoma Classification. An antigen-driven primary pulmonary lymphoproliferative disorder is suggested. However, despite bone marrow and lung explant analysis, no patient had histological evidence of lymphoma.

In light chain amyloidosis, the tropism of organ involvement depends on the variability of the gene encoding for the monoclonal Ig light chain variable region [7]. However, we have no relevant data concerning LCDD.

In several case reports, the treatment for CL-LCDD associated with chronic respiratory insufficiency is lung transplantation, with a maximum follow-up of 4 years without recurrence. One patient received chemotherapy and autologous peripheral blood stem cell transplantation before lung transplantation but with an increased number of lung cysts [1]. Because the patients presented only lung disease and did not have lymphoma, lung transplantation was appropriate.

In systemic LCDD, monoclonal plasmacytic dyscrasia is frequent, and multiple myeloma, Waldenstrom's macroglobulinaemia, lymphoma and CLL may be involved. Unlike treatment for local disease, treatment for systemic LCDD aims to reduce the sFLC level to limit the toxicity of non-amyloid deposits. With management for light chain amyloidosis, chemotherapy can be associated with peripheral blood stem cell transplantation [8].

In our case, clinical and paraclinical investigations revealed cervical and mediastinal lymphadenopathy, a monoclonal IgM peak and a marked increase in $\kappa$-sFLC level, all suggestive of lymphoma, which was found in the lymph nodes and in bone marrow. Autologous peripheral blood stem cell transplantation was performed because of the respiratory and haematological relapse after the two lines of chemotherapy, with increased $\kappa$-sFLC level. This treatment has been shown to be efficienct in light chain amyloidosis [9] and, more recently, in lymphoplasmacytic lymphoma [10]. Our case of CL-LCDD achieved respiratory stability and a haematological response, with a marked decrease in $\kappa$-sFLC level maintained over time. The consensus opinion from the 10th International Symposium on Amyloid and Amyloidosis [11] contains no clear definition of a pulmonary response to treatment. An improvement in interstitial disease is rare. A $50 \%$ reduction in sFLC level is defined as a partial haematological response. DisPenZIERI et al. [12] suggested that the low absolute level of sFLC seems to be the best predictor of overall survival after peripheral blood stem cell transplantation and organ response, rather than a percentage reduction.

Our case of CL-LCDD differs from other cases by the evidence of lymphoma and treatment choice. We are cautious because of the worsening CT evidence of cystic lesions. However, peripheral blood stem cell transplantation was efficient in ameliorating the haematological disorder and stabilising the clinical respiratory condition. As in systemic LCDD with end-stage renal disease, organ transplantation may be considered after long-term reduction of the sFLC level, but relapse is a risk [13].

We report the first case of CL-LCDD revealing a lymphoplasmacytic lymphoma with positive staining for IgM $\kappa$ that was treated with autologous peripheral blood stem cell transplantation, after inefficient chemotherapy.

@ERSpublications

We report a case of CL-LCDD that was treated with autologous peripheral blood stem cell transplantation http://ow.ly/NUQOt

Aurélie Le Borgne ${ }^{1}$, Grégoire Prévot ${ }^{1}$, Isabelle Rouquette ${ }^{2}$, Anne Huynh ${ }^{3}$, Laurent Têtu ${ }^{1}$, Fabrice Projetti ${ }^{2}$, Miguel Carreiro ${ }^{4}$, Raphaël Borie ${ }^{5}$, Arnaud Jaccard ${ }^{6}$, Christian Recher ${ }^{3}$ and Alain Didier ${ }^{1}$

${ }^{1} \mathrm{CHU}$ Toulouse, Service de pneumologie, Toulouse, France. ${ }^{2} \mathrm{CHU}$ Toulouse, Service d'anatomopathologie, Toulouse, France. ${ }^{3} \mathrm{CHU}$ Toulouse, Service d'hématologie, Toulouse, France. ${ }^{4} \mathrm{CH}$ Montauban, Service d'hématologie et oncologie, Montauban, France. ${ }^{5}$ Hopital Bichat, Pneumologie A, Paris, France. ${ }^{6}$ CHU Limoges - Service d'Hématologie et de Thérapie Cellulaire, Limoges, France.

Correspondence: Aurélie Le Borgne, Larrey Hospital Respiratory Medicine, 24 chemin de pouvourville, TSA 30030, Toulouse 31059, Cedex 9, France. E-mail: aurelie.e.leborgne@gmail.com

Received: March 262014 | Accepted after revision: May 132015 | First published online: July 092015

Conflict of Interest: None declared.

\section{References}

1 Colombat M, Stern M, Groussard O, et al. Pulmonary cystic disorder related to light chain deposition disease. Am J Respir Crit Care Med 2006; 173: 777-780.

2 Colombat $\mathrm{M}$, Gounant $\mathrm{V}, \mathrm{Mal} \mathrm{H}$, et al. Light chain deposition disease involving the airways: diagnosis by fibreoptic bronchoscopy. Eur Respir J 2007; 29: 1057-1060. 
3 Girard N, Vasiljevic A, Cottin V, et al. Respiratory failure with diffuse bronchiectases and cryoglobulinaemia. Eur Respir J 2008; 31: 1374-1378.

4 Khoor A, Myers JL, Tazelaar HD, et al. Amyloid-like pulmonary nodules, including localized light-chain deposition: clinicopathologic analysis of three cases. Am J Clin Pathol 2004; 121: 200-204.

5 Luraine R, Sohier L, Kerjouan M, et al. Une cause rare de maladie kystique pulmonaire : maladie à dépôts de chaînes légères d'immunoglobuline [An unusual cause of cystic lung disease: light chain deposition disease]. Rev Mal Respir 2013; 30: 567-571.

6 Colombat M, Mal H, Copie-Bergman C, et al. Primary cystic lung light chain deposition disease: a clinicopathologic entity derived from unmuted B cells with a stereotyped IGHV4-34/IGKV1 receptor. Blood 2008; 112: $2004-2012$.

7 Abraham RS, Geyer SM, Price-Troska TL, et al. Immunoglobulin light chain variable (V) region genes influence clinical presentation and outcome in light chain-associated amyloidosis (AL). Blood 2003; 101: 3801-3808.

8 Guidelines Working Group of UK Myeloma Forum, British Commitee for Standards in Haematology, British Society for Haematology. Guidelines on the diagnosis and management of AL amyloidosis. Br J Haematol 2004; 125: 681-700.

9 Dispenzieri A, Kyle RA, Lacy MQ, et al. Superior survival in primary systemic amyloidosis patients undergoing peripheral blood stem cell transplantation: a case-control study. Blood 2004; 103: 3960-3963.

10 Dimopoulos MA, Gertz MA, Kastritis E, et al. Update on treatment recommendations from the Fourth International Workshop on Waldenstrom's macroglobulinemia. J Clin Oncol 2009; 27: 120-126.

11 Gertz MA, Comenzo R, Falk RH, et al. Definition of organ involvement and treatment response in immunoglobulin light chain amyloidosis $(\mathrm{AL})$ : a consensus opinion from the 10th International Symposium on Amyloid and Amyloidosis, Tours, France, 18-22 April 2004. Am J Hematol 2005; 79: 319-328.

12 Dispenzieri A, Lacy MQ, Katzmann JA, et al. Absolute values of immunoglobulin free light chains are prognostic in patients with primary systemic amyloidosis undergoing peripheral blood stem cell transplantation. Blood 2006; 107: 3378-3383.

13 Leung N, Lager DJ, Gertz MA, et al. Long-term outcome of renal transplantation in light-chain deposition disease. Am J Kidney Dis 2004; 43: 147-153.

\section{Reversal of central sleep apnoea with change from methadone to buprenorphine-naloxone: a case report}

\section{To the Editor:}

Preventing prescription opioid poisoning deaths is a major public health priority in Western societies. Deaths from these medications exceed deaths from all illicit drugs combined [1]. Methadone (for pain treatment) is involved in one third of US prescription opioid overdose deaths despite accounting for only $5 \%$ of dispensed opioids [2]. There is a dose-dependent increase in the severity of central sleep apnoea (CSA) with methadone [3-5] and sleep disordered breathing is a contributing factor in methadone-related deaths [2]. The partial $\mu$-agonist buprenorphine is putatively safer than methadone with a ceiling effect upon respiratory depression [6]. However, the effect of buprenorphine on breathing during sleep remains unclear. The only relevant report from a cross-sectional observational study suggested that buprenorphine-naloxone therapy may induce significant CSA and hypoxaemia [7].

In contrast, we demonstrate a case of significant CSA reversed following a change from methadone to buprenorphine-naloxone therapy, together with improved hypoxaemia and normalised awake ventilatory control. A 47-year-old man who had been receiving methadone treatment for 3 years and with a 15-year of intravenous heroin use history was referred for overnight polysomnography (PSG). The patient had symptoms of snoring, witnessed sleep apnoeas and daytime sleepiness with an Epworth Sleepiness Scale (ESS) score of 11. During methadone treatment, he described frequent nausea, occasional vomiting, reduced libido and lack of motivation. He had no history of cardiac or neurological disease. His body mass index was $26 \mathrm{~kg} \cdot \mathrm{m}^{-2}$. He reported no recent substance use (confirmed by regular urinalysis over the preceding month) except from smoking $\sim 1 \mathrm{~g}$ cannabis per day. His oral methadone dose was $47.5 \mathrm{mg}$ once daily. Approximate peak plasma ( $2 \mathrm{~h}$ after dosing) [8] level of R-methadone was $184 \mathrm{ng} \cdot \mathrm{mL}^{-1}$ and $\mathrm{S}$-methadone was $213 \mathrm{ng} \cdot \mathrm{mL}^{-1}$. Olanzapine $10 \mathrm{mg}$ nocte was his only other medication, prescribed for anxiety by his general practitioner. His awake hypercapnic ventilatory response (HCVR) and hypoxic ventilatory response (HVR) was measured using a validated method [9]. 